\title{
Experiencias activas, espacios pasivos. Una propuesta de Educación Artística contemporánea en espacios tradicionales.
}

\section{Active experiences, passive spaces. A proposal for contemporary Artistic Education in traditional spaces.}

\section{Inés Fombella Coto}

Universidad de Oviedo (España)

fombellaines@uniovi.es
Recibido: 15/09/2020 Revisado: 18/10/2020

Aceptado: 09/12/2020 Publicado: 14/12/2020

\section{Resumen}

El espacio educativo ha permanecido invariable durante el último siglo, respondiendo a un sistema organizativo propio de un paradigma educativo obsoleto, mientras que las metodologías docentes han ido avanzando hacia un enfoque activo. Si bien este anacronismo entre práctica docente y espacio educativo es generalizado, en el caso de la Educación Artística contemporánea la falta de correspondencia resulta especialmente acusada.

Desde la asignatura de Expresión Plástica y su Didáctica del Grado de Maestro y Maestra en Educación Primaria de la Universidad de Oviedo se plantea, dentro de la formación inicial de los futuros docentes, despertar su consciencia y desarrollar su sensibilidad hacia el espacio físico en que llevará a cabo su labor profesional como parte de su aprendizaje.

\section{Sugerencias para citar este artículo:}

Fombella Coto, Inés (2020). Experiencias activas, espacios pasivos. Una propuesta de Educación Artística contemporánea en espacios tradicionales. Tercio Creciente (Monográfico extraordinario IV), (pp. 47-58), https://dx.doi.org/10.17561/rtc.extra4.5767

FOMBELLA COTO, INÉS. Experiencias activas, espacios pasivos. Una propuesta de Educación Artística contemporánea en espacios tradicionales. Tercio Creciente (Monográfico extraordinario IV), diciembre 2020, pp. 47-58, https://dx.doi.org/10.17561/rtc.extra4.5767 
A través de propuestas artístico educativas en las que el espacio supone un factor fundamental a nivel estético, estructura y conceptual, el alumnado experimenta las posibilidades del ambiente físico, lo que repercutirá en su futura práctica docente.

\section{Abstract}

While teaching methodologies have been moving towards an active approach, the educational space has remained unchanged over the last century, in response to an organizational system that is part of an obsolete educational paradigm. Although this anachronism between teaching practice and educational space is prevalent in the case of contemporary Artistic Education, the lack of correspondence is particularly noticeable.

From the course of Didactic of Arts in the Bachelor's Degree in Primary Education of the University of Oviedo, it is proposed, within the initial training of future teachers, to awaken their conscience and develop their sensitivity towards the physical space in which they will carry out their professional work as part of their learning.

Through artistic educational proposals in which the space is a fundamental factor on an aesthetic, structural and conceptual level, students will experience the possibilities of the physical environment, which will have an impact on their future teaching practice.

\section{Palabras clave}

Educación artística/espacio de aprendizaje/espacio educativo/escenario de aprendizaje

\section{Keywords}

Arts Education/Learning Space/Educational Space/Learning Landscape

\section{Sugerencias para citar este artículo:}

Fombella Coto, Inés (2020). Experiencias activas, espacios pasivos. Una propuesta de Educación Artística contemporánea en espacios tradicionales. Tercio Creciente (Monográfico extraordinario IV), (pp. 47-58), https://dx.doi.org/10.17561/rtc.extra4.5767

FOMBELLA COTO, INÉS. Experiencias activas, espacios pasivos. Una propuesta de Educación Artística contemporánea en espacios tradicionales. Tercio Creciente (Monográfico extraordinario IV), diciembre 2020, pp. 47-58, https://dx.doi.org/10.17561/rtc.extra4.5767 


\section{Introducción}

La educación atraviesa, a todos los niveles y en todas las etapas educativas, un proceso de transformación hacia un modelo más centrado en el alumnado como protagonista de su aprendizaje. Así, la tendencia es ir dejando atrás el modelo educativo tradicional basado en la instrucción directa y el papel pasivo de los y las estudiantes. De este modo, un paradigma basado en un aprendizaje significativo, en las experiencias directas, en el desarrollo de capacidades por encima de la memorización de contenidos, se abre paso como respuesta acorde a las demandas actuales de la sociedad (Mingorance, Trujillo, Cáceres y Torres 2017).

En este contexto en evolución, la Educación Artística contemporánea se esfuerza por superar una tradición academicista resultante en una visión distorsionada de la enseñanza de las artes visuales y plásticas que aún impera en muchos centros educativos. Para modificar la experiencia basada en el desarrollo de habilidades técnicas, o en la simple realización de manualidades sin objetivos artísticos, se avanza en la integración de nuevos medios sobre los que explorar, de nuevos formatos que acerquen el arte contemporáneo al alumnado.

Dentro de este proceso, cada vez más acusado y enmarcado en el cambio de paradigma educativo a nivel global, podemos influir como docentes en las experiencias artístico educativas que nuestro alumnado universitario, futuro profesorado en formación, vivirá mediante el diseño de propuestas basadas en metodologías activas acordes al aprendizaje significativo que hoy se demanda.

Sin embargo, cabe preguntarse por la dimensión física y características ambientales de los lugares donde se llevan a cabo esas experiencias. ¿Cómo son los espacios donde se enseña educación artística? ¿Cómo deberían ser? ¿Qué necesidades espaciales y ambientales demanda una Educación Artística contemporánea basada en la experimentación estética, sensorial, corporal? ¿Podemos crear experiencias educativas activas en espacios pasivos? 


\section{Espacios pasivos: la importancia de la arquitectura en la educación}

La mayoría de los emplazamientos en que se imparten clases actualmente responden a un tipo de organización espacial resultante de un sistema educativo basado en el docente como única fuente de información (Escofet, Gros, López y Marimón-Martí, 2019).

Estos espacios se caracterizan por poseer una planta de forma rectangular, en uno de cuyos lados largos suele situarse las ventanas y la puerta en el opuesto. En los lados cortos, uno resulta el principal al ser donde habitualmente se sitúa la pizarra y la mesa del docente.

Los pupitres del alumnado se disponen en filas y columnas dejando estrechos pasos entre ellos, formando una retícula. De esta forma, el esquema resultante promueve un tipo de organización estática y axial, en la que el foco se sitúa en la dirección en que se orientan las mesas, coincidente con la posición del docente.

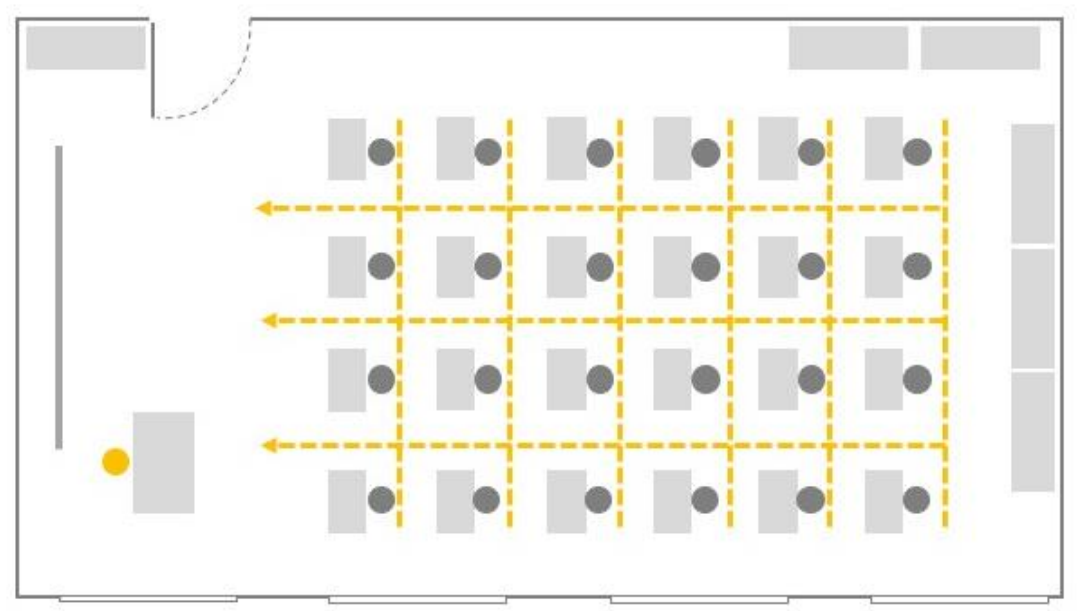

Figura 1. Esquema aula tradicional. Elaboración propia.

Este esquema, que resulta tan familiar y se encuentra tan extendido, dificulta la interacción entre el alumnado, así como con el profesorado, favoreciendo una 
comunicación unidireccional en la que el alumnado, irremediablemente, debe adoptar un papel pasivo (Picó, 2016).

Pese a que han sido muchas las teorías y movimientos pedagógicos que, desde finales del siglo XIX, han apostado por un sistema organizativo más flexible $y$ en consonancia con un aprendizaje más activo (Atrio, Raedó y Navarro, 2016) el aula tradicional, con sus filas de pupitres, con sus alumnos y alumnas alineados mirando en la misma dirección, sigue imperando en centros educativos de diferentes etapas. De este modelo se conserva, además de la organización espacial, la asociación de orden con disciplina, y de unidireccionalidad con atención (Bonastra, Farrero, Jové, y Llonch, 2014).

Desde el kindergarten de Friedrich Fröbel a principios del siglo XIX, al modelo Waldorf o la pedagogía Montessori a mediados del siglo XX, pasando por Reggio Emilia y la Institución Libre de Enseñanza, diferentes modelos pedagógicos han recogido en sus postulados el espacio educativo como factor fundamental en el aprendizaje.

Durante la última década, especialmente, este tipo de modelos pedagógicos han experimentado una revisión y valoración de sus principios, motivando un cambio que paulatinamente va calando entre el tejido educativo de diferentes etapas y diferentes países. Este resurgimiento de las llamadas pedagogías activas, que responde a un cuestionamiento del modelo educativo tradicional, conlleva además la puesta en valor de la idea de que el espacio en que se desarrolla posee una gran influencia.

Además, desde diferentes campos de conocimiento como la psicología ambiental o la neuroarquitectura, numerosos estudios arrojan evidencias sobre la influencia que el espacio construido tiene en el proceso de enseñanza y aprendizaje, siendo fundamental tanto en el bienestar, como el comportamiento y rendimiento del alumnado y también del profesorado (Picó, 2016).

Pese a ello, durante décadas el espacio ha sido considerado como un factor invariable, sin ser considerado como un recurso educativo. El profesorado no lo advierte como un factor relevante dentro de su práctica, ni se considera un gestor de este, lo que perpetúa un modelo organizativo que responde a un sistema educativo de otra época (Palacios, 2015). El docente o la docente, por norma general, no actúa sobre el espacio que le rodea, no modifica la disposición de los elementos que están dentro del aula, desaprovechando así un valioso recurso didáctico. 
En el caso de la Educación Artística, además, este desaprovechamiento implica la ausencia de propuestas artístico educativas basadas en el movimiento, en la exploración del espacio, en el volumen, en dinámicas cambiantes... dejando fuera del aula referencias del mundo de la instalación artística o la performance y metodologías artísticas de enseñanza.

Así, algunos de los factores que influyen en esta situación, pueden ser:

1. La falta de sensibilidad hacia el espacio en general y educativo en particular.

2. El desconocimiento de la importancia e impacto que el ambiente construido tiene tanto sobre el rendimiento como el bienestar de sus ocupantes, en este caso, alumnado y profesorado.

3. La inmovilidad imperante de las aulas, advirtiéndolas como elementos imperturbables, desaprovechando así la oportunidad de emplearlas como recurso educativo y artístico.

4. La falta de formación inicial en los diferentes grados de las facultades de Formación del Profesorado y Educación sobre el uso del espacio como recurso educativo y su influencia en el proceso de enseñanza y aprendizaje.

\section{Una educación artística contemporánea en la formación del profesorado}

El alumnado universitario de la facultad de Formación del Profesorado y Educación se prepara para desarrollar en el futuro su profesión en un contexto educativo en transformación. Este cambio de paradigma se implementa, en la mayoría de los casos, en edificios diseñados para responder a un modelo educativo tradicional, basado en el docente como centro del proceso de enseñanza y no en el alumnado como protagonista de su aprendizaje. Como actual alumnado y futuro profesorado, necesitan desarrollar en su formación inicial una visión crítica sobre el espacio que les rodea y sobre las cualidades ambientales de los recintos en que llevan a cabo diferentes actividades y modalidades de trabajos.

En la asignatura Expresión Plástica y su Didáctica del Grado en Maestro de Educación Primaria de la Universidad de Oviedo, además de tener como principal objetivo la superación de ideas obsoletas sobre la Educación Artística, se trabaja desde 
el cambio hacia un modelo contemporáneo, lo que requiere la adquisición por parte del alumnado de una consciencia del espacio en que desarrollarán su labor docente en el futuro, dotándoles así de sensibilidad hacia el entorno.

Desde nuestra propia práctica como docentes, observamos que la Educación Artística contemporánea parece no encajar en un aula tradicional. Un ambiente lleno de mobiliario, sin espacio libre que permita moverse o desplazarse por el aula dificulta generar experiencias artísticas basadas en un enfoque activo del aprendizaje. Así, como educadores y educadoras artísticas nos cuestionamos ¿es posible proponer dinámicas basadas en enfoques activos en estos contenedores pasivos? ¿Tiene cabida la Educación Artística contemporánea en un aula tradicional?

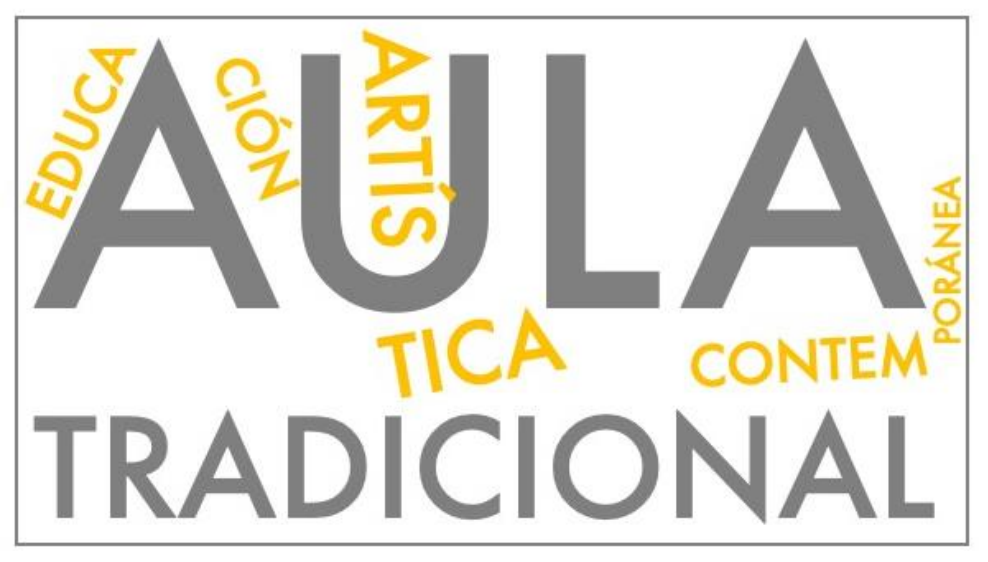

Figura 2. Esquema: educación artística en aula tradicional. Elaboración propia.

Si se opta por una concepción tradicional de la Educación Plástica consistente en realización de ejercicios clásicos, con explicaciones magistrales, propuestas basadas en manualidades, no tiene ninguna dificultad llevarla a cabo en las aulas actuales. Así, podemos proponer a nuestro alumnado ejercicios que se realicen en sus mesas, sin moverse, con lo que el espacio no tendrá mucha importancia.

Sin embargo, en una visión contemporánea de la labor como docentes de Educación Artística, partimos de la base de una concepción de la asignatura basada en el desarrollo de la percepción, que fomente la experimentación, la curiosidad, el análisis y la formulación de preguntas, que eduque la mirada, que fomente la interacción, que implique un enfoque activo, que genere creación y colaboración. Esto es, una idea de la 
Educación Artística basada en generar dinámicas activas, basada en diseñar experiencias educativas que estimulen los sentidos, basada en una educación estética.

Si esto tenemos que llevarlo a cabo en aulas tradicionales, ¿de qué herramientas disponemos? ¿Cómo podemos hacer que nuestro alumnado universitario, futuro profesorado de primaria, cuestione cómo debe ser la Educación Artística contemporánea y, a la vez, reflexione sobre la importancia del uso del espacio educativo? ¿Cómo hacer que nuestro alumnado especule y encuentre soluciones para generar experiencias artístico educativas contemporáneas en un aula tradicional?

\section{Experiencias activas: herramientas para un uso activo del espacio}

El objetivo principal se plantea como provocar que el alumnado universitario de la asignatura de Expresión Plástica y su Didáctica, futuro profesorado de Educación Primaria, se cuestione cómo debe ser la Educación Artística contemporánea y, a la vez, reflexione y adquiera consciencia sobre la importancia del uso del espacio educativo. ¿Cómo podemos llegar a cumplir este objetivo? Proponiendo a nuestros y nuestras estudiantes experiencias educativas en las que el espacio sea protagonista, varíe de una sesión a otra, sea generador de dinámicas. De ese modo podrán empezar a ser conscientes del impacto que tiene el ambiente construido tanto en el aprendizaje como en la práctica docente. Comenzarán a ver el ambiente como un recurso didáctico, y a través de los escenarios propuestos, también como un recurso artístico. Con el tiempo, podrán romper la barrera de sentirse gestores del espacio en que hoy estudian y en un futuro trabajarán.

Para ello, en primer lugar, con respecto al uso del espacio, es importante que nuestro alumnado sea consciente del tipo de entornos en que se imparte enseñanza y, además, del efecto que estos tienen sobre los usuarios. En segundo lugar, es necesario generar reflexión sobre el uso del espacio, para crear así un sentido crítico a este respecto que les permita cuestionar el aula tradicional. Pero, para ello, debemos ofrecerles herramientas que les permitan afrontar la tarea de actuar sobre su entorno para integrarlo en su práctica docente. Así, podrán llegar a generar propuestas artístico educativas basadas en un enfoque docente contemporáneo y activo, en las que el espacio sea una ayuda y no un obstáculo. 
A fin de que empiecen a ser conscientes de las posibilidades que el espacio físico posee, podemos instarles observar su entorno inmediato, hacerles reparar en esos elementos clásicos aparentemente fijos como el mobiliario, el tipo de organización espacial o la morfología del aula que conforman un aula tradicional. Lo más evidente y fundamental, es hacer ver a nuestro alumnado que estos elementos, usualmente asumidos como inamovibles, en la mayoría de los casos no lo son, y que, concibiendo el aula como un escenario de aprendizaje, hay cambios sencillos que pueden suponer una gran diferencia tanto a nivel estético como metodológico.

Llevando a cabo propuestas didácticas en las que estos elementos modifiquen su disposición tradicional, varíen de una clase a otra, y se empleen de formas diversas, se implantará la idea de movilidad, asumiendo como parte de su labor docente el empleo de mobiliario y accesorios del aula de la forma óptima en función del tipo de dinámica a realizar.

Como recurso no solo didáctico, sino artístico, la liberación del espacio mediante el desplazamiento de elementos móviles, permite generar dinámicas activas que fomenten el movimiento y propuestas, como instalaciones artísticas o performances, difíciles de ejecutar en un espacio tradicional copado de mesas y sillas.

Además de reorganizar el aula y de aprovechar los elementos móviles en las propuestas educativas, hay actos tan sencillos como apagar la luz que pueden modificar por completo la percepción de un ambiente. ¿Qué sucede si el aula está de repente a oscuras? ¿Y si se encienden unos focos aquí y allí? ¿Qué pasa si jugamos con las sombras, si experimentamos cómo vemos las cosas con diferentes gradientes lumínicos? ¿Y si dejamos entrar la luz natural poco a poco?

El manejo de la luz como recurso estético y didáctico puede ayudar a generar ambientes muy diferentes en un aula tradicional, generando soluciones variables, que limiten o potencien la visión sobre determinados elementos. Experimentar con la luz, permitirá al futuro profesorado ser consciente del impacto que la ausencia o focalización de esta puede aportar a sus propuestas. Desde un punto de vista artístico, les permitirá desarrollar su sentido estético y aumentar su sensibilidad hacia factores ambientales. 


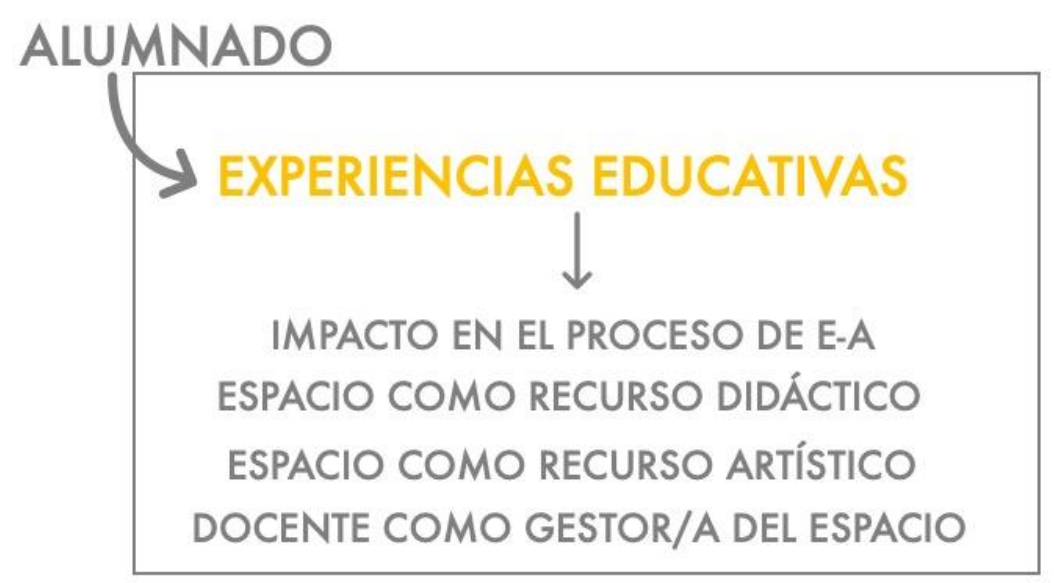

Figura 3. Esquema del proceso. Elaboración propia.

A modo de resumen, todo comienza ofreciendo a nuestro alumnado, futuros maestros y maestras de Educación Primaria, experiencias artístico educativas que les permitan experimentar el espacio de una forma diferente a la tradicional, jugando con elementos como la iluminación, la organización, los materiales empleados, la estética, etc. Al ampliar sus conocimientos sobre gestión del espacio y generación de escenarios de aprendizaje, lo que ellas y ellos experimentan como alumnado, podrán ponerlo en práctica como profesorado. Así, proporcionarán a su futuro alumnado experiencias artísticas basadas en la educación estética, diseñadas desde una concepción contemporánea de la Educación Artística y con el espacio como herramienta fundamental.

\section{Conclusiones}

Aunque el espacio educativo es un factor determinante en la práctica educativa, actualmente se encuentra desaprovechado por décadas de normalización de un esquema organizativo obsoleto. Es necesario que, desde la formación inicial del profesorado, se fomente la sensibilidad hacia el ambiente construido, hacia el entorno que rodea al docente $y$ al discente, al ser un elemento fundamental en el proceso de enseñanza y aprendizaje.

A través de acciones educativas y artísticas, en las que el espacio constituye un recurso didáctico, y de sesiones dedicadas a la reflexión sobre el espacio educativo, se 
deben plantear cuestiones como ¿de qué recursos disponemos para modificar el espacio? ¿Qué necesidades tendremos a la hora de elaborar propuestas artístico educativas desde un punto de vista contemporáneo?

No se trata de presentar un catálogo de soluciones organizativas que el alumnado pueda reproducir, sino de generar experiencias, durante su formación inicial, que les permitan tomar consciencia y reflexionar sobre:

1. El impacto que el espacio educativo tiene en el proceso de enseñanza y aprendizaje tanto en el alumnado como en el profesorado.

2. Cuáles son los elementos físicos y ambientales que influyen en el proceso de enseñanza y aprendizaje.

3. La relevancia, por tanto, que el ambiente construido tiene en su práctica docente, pudiendo convertirse en un valioso recurso educativo.

4. La importancia que, como recurso artístico, puede tener el espacio físico en el desarrollo de propuestas educativas.

Cada estudiante y futuro docente, al igual que lo hará con las metodologías que le sean presentadas durante su formación, aplicará aquello que se adapte a su enfoque educativo personal, que se adecúe a su forma de realizar su trabajo y que le sea útil para llevar a cabo sus propuestas. Y para ello su formación inicial debe proveerle de este aprendizaje.

\section{Referencias}

Atrio, S., Raedó, J., \& Navarro, V. (2016). Educación y Arquitectura: ayer, hoy, mañana. Crónica del III Encuentro Internacional de Educación en Arquitectura para la Infancia y la Juventud. Tarbiya, revista de Investigación e Innovación Educativa, (44), 131-148. Disponible en https://revistas.uam.es/tarbiya/article/view/6809

Bonastra, Q., Farrero, M., Jové, G., \& Llonch, N. (2014). Arte, arquitectura y docencia. Los espacios de libertad. En el cubo blanco y la disciplina. Scripta Nova, 18(493), 1-31. Disponible en http://hdl.handle.net/10459.1/48731

Escofet, A., Gros, B., López, M. \& Marimon-Martí, M. (2019). Percepción del profesorado sobre la integración de la tecnología en el espacio escolar. RIITE. Revista 
Interuniversitaria de Investigación en Tecnología Educativa, 6, 37-47. doi: https://doi.org/10.6018/riite.360631

Mingorance, A.C., Trujillo, J.M., Cáceres, P., \& Torres, C. (2017). Mejora del rendimiento académico a través de la metodología de aula invertida centrada en el aprendizaje activo del estudiante universitario de ciencias de la educación. Journal of Sport and Health Research, 9(1), 129-136. Disponible en https://bit.ly/20eppGj

Palacios, A. (2015). Inventando mundos. Diálogos creativos con David Gamella. Alcalá de Henares: Centro Universitario Cardenal Cisneros.

Picó, J. (2016). Un profesor llamado espacio. Relacionando los espacios de aprendizaje y las metodologías educativas del SXX. Revista ruta maestra, 17. 